\title{
'Splenic switch-off' to detect adenosine understress; a novel method to improve test sensitivity
}

Charlotte Manisty ${ }^{1 *}$, David P Ripley ${ }^{4}$, Gaby Captur $^{3}$, Charles Peebles $^{2}$, Timothy C Wong $^{5}$, Erik B Schelbert ${ }^{5}$, Anna S Herrey ${ }^{6}$, John P Greenwood ${ }^{4}$, James Moon ${ }^{3}$

From 17th Annual SCMR Scientific Sessions

New Orleans, LA, USA. 16-19 January 2014

\section{Background}

The sensitivity of adenosine perfusion CMR is reduced by false negative scans, with up to $50 \%$ resulting from inadequate pharmacological stress. Without a robust physiological marker for adequate myocardial hyperaemia, this false negative rate is difficult to address. We observed that splenic perfusion is markedly attenuated with adenosine - compared both to rest and to myocardial perfusion. In this collaborative multi-center study, we investigate the pharmacology of 'splenic switch-off', and evaluate its potential clinical utility as a marker of inadequate stress in adenosine perfusion imaging.

\section{Methods}

We assessed splenic perfusion in 4 cohorts acquired in 4 separate CMR units using 3 different pharmacological stressors. This study included: • Verification cohort of 50 adenosine perfusion scans (London, UK); to determine
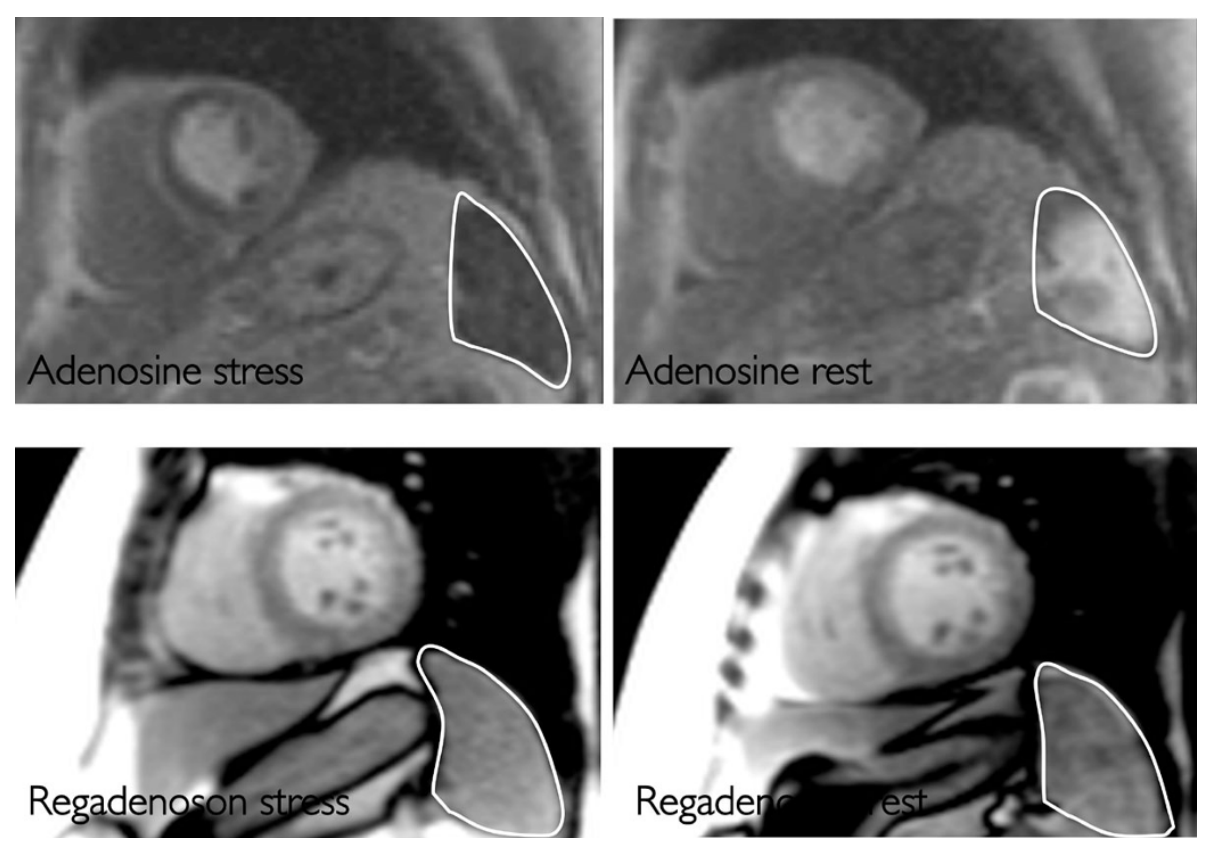

Figure 1 Splenic perfusion at stress and rest with adenosine (upper panels) and regadenoson (lower panels), showing splenic switchoff with adenosine only.

'Heart Hospital Imaging Centre and Imperial College, London, UK

Full list of author information is available at the end of the article

(c) 2014 Manisty et al.; licensee BioMed Central Ltd. This is an Open Access article distributed under the terms of the Creative Commons 


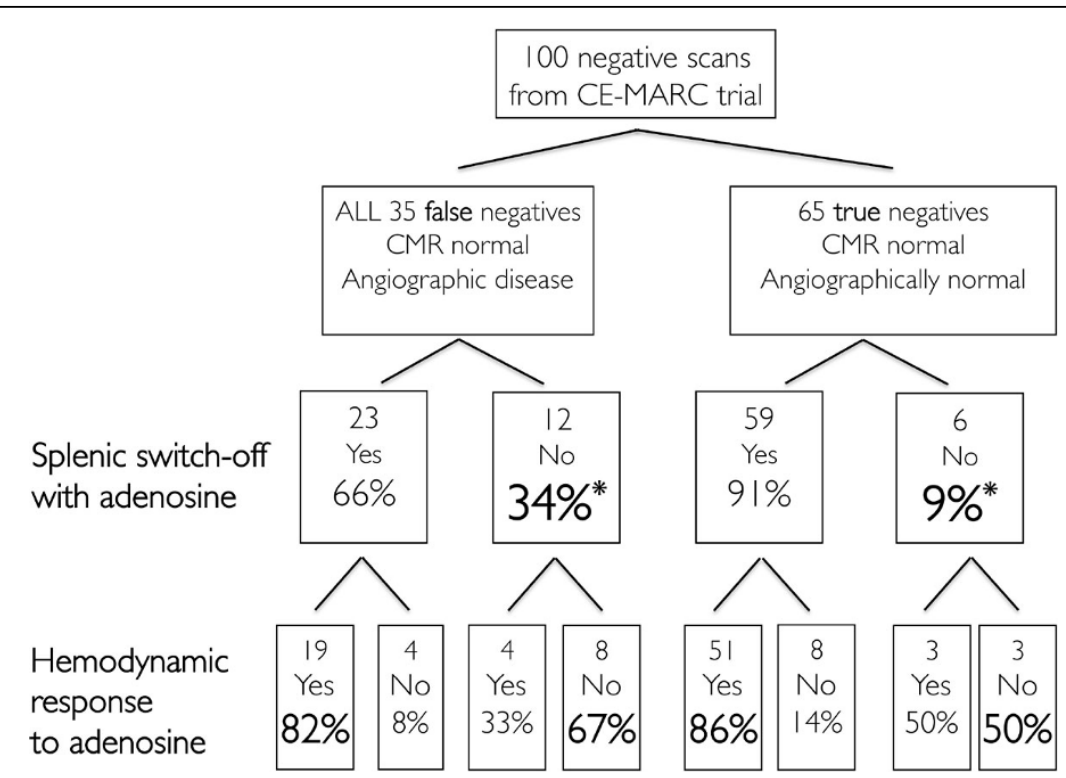

Figure 2 Data from CE-MARC trial to assess splenic and hemodynamic responses to adenosine There were significantly more patients with false negative CMR perfusion scans who failed to switch-off splenic perfusion with adenosine (indicating inadequate pharmacological stress) in comparison to those with true negative scans. Concordance was good between hemodynamic and splenic responses to adenosine. ${ }^{*} p=0.0027$.

if splenic perfusion is consistently switched-off with adenosine. 2 comparison cohorts using alternative pharmacological stressors (25 dobutamine scans; Southampton, UK and 25 regadenoson scans; Pittsburgh, USA); to assess whether generic stress (or only adenosine) causes splenic switch-off. - Clinical utility cohort of 100 adenosine scans (35 false and 65 true negative) from the CE-MARC trial (Leeds, UK); to assess whether failure of splenic switch-off could be a useful clinical indicator of inadequate stress.

\section{Results}

The spleen was visible in $98.5 \%$ of scans and grading of splenic perfusion was concordant between 2 blinded observers, $\kappa=0.84$. Splenic switch-off occurred in $92 \%$ of adenosine studies acquired in London, but did not occur either with dobutamine or regadenoson perfusion studies, Figure 1. Measuring perfusion semi-quantitatively using signal intensity, splenic perfusion with adenosine stress was significantly lower than at rest $(8.1 \pm 9$ versus $33.3 \pm 19$ arbitrary units, $\mathrm{p}<0.0001)$, in contrast to with regadenoson where it increased significantly $(123.7 \pm 56.7$ versus $144.6 \pm$ $59.2 \mathrm{au}, \mathrm{p}=0.003$ ). With dobutamine (where only stress images were acquired), splenic perfusion was greater than myocardial $(54.1 \pm 1$ versus $67.6 \pm 25.2 \mathrm{au}, \mathrm{p}=0.0005)$, again in contrast to adenosine. Within the CE-MARC cohort, patients with false negative CMR scans had a $36 \%$ rate of failed splenic switch-off. By contrast, the true negative group had a $9 \%$ rate ( $p=0.0027$ for difference), Figure 2 . Splenic response to adenosine was concordant with haemodynamic response in $81 \%$ of subjects.

\section{Conclusions}

Splenic switch-off with adenosine is a new observation, and although a drug-specific effect, can be assessed in nearly all scans. Rescanning individuals with failure of splenic switch-off would reduce false negative scans by a third, but it may be that up to 1 in 11 of all adenosine perfusion patients are understressed. Further work is needed on this important sign.

\section{Funding}

$\mathrm{CM}$ is an NIHR Clinical Lecturer.

\section{Authors' details}

${ }^{1}$ Heart Hospital Imaging Centre and Imperial College, London, UK. ${ }^{2}$ University Hospital, Southampton, Southampton, UK. ${ }^{3}$ Heart Hospital Imaging Centre and University College, London, UK. ${ }^{4}$ Multidisciplinary Cardiovascular Research Centre (MCRC) \& Leeds Institute of Genetics, Health and Therapeutics, University of Leeds, Leeds, UK. ${ }^{5}$ UPMC Cardiovascular Magnetic Resonance Center, Heart and Vascular Institute, University of Pittsburgh, Pittsburgh, Pennsylvania, USA. ${ }^{6}$ Heart Hospital Imaging Centre and Royal Free Hospital, London, UK.

Published: 16 January 2014

doi:10.1186/1532-429X-16-S1-01

Cite this article as: Manisty et al:: 'Splenic switch-off' to detect adenosine understress; a novel method to improve test sensitivity. Journal of Cardiovascular Magnetic Resonance 2014 16(Suppl 1):O1. 